\title{
Metabolic Perturbations and Severe COVID-19 Disease: Implication of Molecular Pathways
}

\author{
Ersilia Nigro $\left(\mathbb{D},{ }^{1,2}\right.$ Fabio Perrotta $\left(\mathbb{D},{ }^{3}\right.$ Rita Polito $\left(\mathbb{D},{ }^{2}\right.$ Vito D'Agnano, ${ }^{4}$ Filippo Scialò $\left(\mathbb{D},{ }^{4}\right.$ \\ Andrea Bianco ${ }^{\mathrm{D}},{ }^{4}$ and Aurora Daniele $\mathrm{ID}^{1,2}$ \\ ${ }^{1}$ Dipartimento di Scienze e Tecnologie Ambientali Biologiche Farmaceutiche, \\ Università Degli Studi Della Campania "Luigi Vanvitelli", Via G. Vivaldi 42, Caserta 81100, Italy \\ ${ }^{2}$ CEINGE-Biotecnologie Avanzate Scarl, Via G. Salvatore 486, Napoli 80145, Italy \\ ${ }^{3}$ Dipartimento di Medicina e Scienze Della Salute "V. Tiberio", Università Del Molise, Campobasso 86100, Italy \\ ${ }^{4}$ Dipartimento di Scienze Mediche Traslazionali e Chirurgiche, Università Della Campania "L. Vanvitelli”, Napoli 80131, Italy \\ Correspondence should be addressed to Aurora Daniele; aurora.daniele@unicampania.it
}

Received 16 July 2020; Revised 4 November 2020; Accepted 17 November 2020; Published 30 November 2020

Academic Editor: Reinhard Depping

Copyright (C) 2020 Ersilia Nigro et al. This is an open access article distributed under the Creative Commons Attribution License, which permits unrestricted use, distribution, and reproduction in any medium, provided the original work is properly cited.

\begin{abstract}
Coronavirus disease (COVID-19) is caused by SARS-CoV-2 virus, which can result in serious respiratory illnesses such as pneumonia leading to respiratory failure. It was first reported in Wuhan, Hubei, China, in December 2019 and rapidly spread globally, becoming a pandemic in March 2020. Among comorbidities observed in SARS-CoV-2 positive patients, hypertension (68.3\%) and type 2-diabetes (30.1\%) are the most frequent conditions. Although symptoms are highly heterogeneous (ranging from absence of symptoms to severe acute respiratory failure), patients with metabolic-associated diseases often experience worse COVID-19 outcomes. This review investigates the association between metabolic disorders and COVID-19 severity, exploring the molecular mechanisms potentially underlying this relationship and those that are responsible for more severe COVID-19 outcomes. In addition, the role of the main biological processes that may connect metabolic alterations to SARS-CoV-2 infection such as hyperglycemia, immune system deregulation, ACE-2 receptor modulation, and inflammatory response is described. The impact of metabolic disorders on the prognosis of COVID-19 has major implications in public health especially for countries affected by a high incidence of metabolic diseases.
\end{abstract}

\section{Introduction}

Cluster cases of pneumonia of unknown origin were described in December 2019 in Wuhan, Hubei Province, China; the cause was later identified as the novel coronavirus SARS-CoV-2 [1]. Subsequently, the spread of the novel SARS-CoV-2 coronavirus has reached pandemic levels as declared by the World Health Organization (WHO) and still represents a global threat for increased morbidity and mortality. WHO data about the pandemic spread on $2^{\text {nd }}$ November 2020 reported 46,166,182 confirmed cases worldwide since the start of the outbreak, and 1,196,326 deaths in 219 countries [2]. In Italy, 632.092 confirmed cases and $38.190(6 \%)$ deaths have been registered according to the Istituto Superiore di Sanità up to November $2^{\text {nd }}$ [3].
Coronaviruses (CoVs) are a large family of enveloped viruses containing single-stranded positive-sense RNA. Previously, two different CoVs, severe acute respiratory syndrome $\mathrm{CoV}$ (SARS) and Middle East respiratory syndrome (MERS), outbreaks caused significant mortality in 2003 and 2009 [4]. SARS-CoV-2 virus entry is mediated by the viral spike protein that forms homotrimers and binds to human cells [5]. The protein spike (S) is composed of two subunits, $\mathrm{S} 1$ responsible for the binding to the host cell membrane (via receptor) and S2 that determines the fusion of the viral and cellular host membranes $[5,6]$. Successively, host proteases cleave the $S$ protein, irreversibly activating the $S$ protein and the consequent entry of the virus [7]. The symptoms, which usually emerge between 2 and 14 days after infection, are strongly heterogeneous ranging from 
absence or mild symptoms to severe disease and death $[1,4]$. The mechanisms and factors influencing the phenotypic manifestations of SARS-CoV-2 infection remain unclear. General health condition, age, and concomitant morbidities appear to determine clinical manifestation and adverse outcomes for SARS-CoV-2-induced pneumonia [6]. Severe and fatal SARS-CoV-2 infection occurs mainly in the elderly or patients with underlying clinical conditions. Indeed, most hospitalized patients, admitted to intensive care units for severe acute respiratory distress syndrome, exhibit comorbid conditions [8, 9]. Previous SARS-CoV and MERS-CoV epidemics also displayed worse outcomes and challenging management when comorbidities were present [10-12]. Emerging data document that SARS-CoV-2 infection is mainly associated with hypertension and/or metabolic disorders (the most common), cerebrovascular diseases, hepatitis B infections, chronic obstructive pulmonary disease, chronic kidney diseases, and malignancy [13, 14]. Individuals affected by type 2 diabetes and severe obesity $(\mathrm{BMI} \geq 40 \mathrm{~kg} / \mathrm{m} 2)$ are more likely to be infected and are at high risk for complications and death from SARS-CoV-2 infection [15-19].

This review analyzes correlations between metabolic disorders, mainly obesity and type 2 diabetes, and SARSCoV-2 infection looking at the underlying molecular mechanisms involved and their impact on prognosis.

\section{Comorbidities in SARS-CoV-2 Infection}

Initial data on SARS-CoV-2 infection documented that patients with preexisting metabolic and cardiovascular diseases experienced unfavorable outcomes $[8,20]$. The influence of comorbidities in coronaviruses infections has been reported during previous SARS and MERS outbreaks [10, 21-28] (see Table 1). Chronic cardiovascular disorders and diabetes mellitus have been reported as the major comorbidities influencing prognosis during previous coronavirus epidemics [29]. A nationwide analysis on SARS-CoV-2 in China reported a $25.1 \%$ prevalence of at least 1 comorbidity among 1.590 patients tested positive for SARS-CoV-2. The authors found a proportion of $8.2 \%$ of diabetes, $16.9 \%$ of hypertension, and $57.3 \%$ of other cardiovascular diseases [13]. Likewise, comorbidities are highly prevalent among SARS-CoV-2 patients who experience a more severe clinical disease course. In Italy, according to the report of Istituto Superiore Sanità (ISS) based on available data on July $9^{\text {th }}, 2020$, among patients with SARS-CoV-2, the mean number of underlying diseases was 3.3 among 3857 patients dying in hospital. A single comorbidity was found in $13.2 \%$ patients, 2 comorbidities in $19.3 \%, 3$ or more in $64 \%$ patients. Among common comorbidities observed in SARS-CoV-2 positive deceased patients, hypertension (66.2\%), type 2-diabetes (29.8\%), and ischemic heart disease $(27.7 \%)$ are the most frequent conditions $[1,30,31]$. In addition, the overall proportion of hypertension and diabetes seems to be about twofold higher in ICU/severe cases than in non-ICU/severe counterparts [31] and although diabetes may not increase the risk of infection, the coexistence of this comorbidity among SARS-CoV-2 patients results in excess mortality [32].

The complex mechanisms linking hypertension and diabetes interact at different levels resulting in bidirectional interplay. Firstly, there are several shared biological mechanisms that influence this multifaceted network; they include unbalanced renin angiotensin aldosterone system homeostasis (RAAS), systemic proinflammatory status, elevated oxidative stress, and increased sympathetic nervous system (SNS) activation [32]. Secondly, perturbation of insulin sensitivity leads to an imbalance in insulin-mediated vasodilator/vasoconstrictor activities. This is associated with vascular stiffness, hypertrophy, fibrosis, and remodeling mainly driven through MAPK dependent signaling pathways. Indeed, insulin stimulates secretion and expression of vasoconstrictor mediators, including endothelin-1, PAI-1, and vascular cell adhesion molecule-1 resulting in increased vasoconstrictor effects [33-35]. Furthermore, obesity and the release of adipose released hormones influence the risk and severity of both hypertension and diabetes [36]. Likewise, both hypertensive status and disproportionate adiposity may alter the interaction between the pulmonary microenvironment, viral pathogenesis, and immune cell trafficking [32, 37].

Emerging data in SARS-CoV-2 indicate the importance of obesity in influencing clinical outcomes. Lighter and coworkers highlighted the impact of obesity in SARS-CoV-2 patients aged $<60$ years. Based on 3.615 subjects tested positive, the authors concluded that patients with a BMI between 30 and 34 were 2.0 (95\% 1.6-2.6, $p<0.0001)$ and 1.8 (95\% CI 1.2-2.7, $p=0.006$ ) times more likely to be admitted to an acute department and ICU, respectively, compared to individuals with a $\mathrm{BMI}<30[28,35]$. However, the comprehensive mechanisms interacting between the virus-induced local inflammation and secondary reactive damage induced from excessive cytokine storm in patients with comorbidities have not been fully clarified.

\section{Molecular Mechanisms Linking Metabolic Diseases and SARS-CoV-2 Infection}

It is plausible that more than one mechanism concurs in determining the relationship between metabolic diseases and SARS-CoV-2 resulting in elevated susceptibility to infection, as well as a worse prognosis of the disease. The interrelations between organs and mechanisms involved are complex; the main factors implicated seem to be as follows: hyperglycemia and immune system deregulation, ACE-2 receptor modulation, and inflammatory response.

3.1. Hyperglycemia, Adipose Tissue, and Immune Response Deregulation. Hyperglycemia has been associated not only with disease severity but also with death in patients with severe SARS-CoV-2 infection [36]. Interestingly, these findings are consistent with studies of patients infected with highly pathogenic avian influenza, as well as SARS and MERS where the presence of uncontrolled hyperglycemia was associated with poor outcomes. Multiple molecular 
TABLE 1: Influence of comorbidities in coronaviruses infections, SARS-CoV, MERS-CoV, and SARS-CoV-2 outbreaks.

\begin{tabular}{lccccc}
\hline & Diabetes (\%) & Hypertension (\%) & HD (\%) & Obesity (\%) & References \\
\hline SARS & $2.47-50.0$ & $4.9-19.4$ & $0.9-32.6$ & - & $18-22$ \\
MERS & $32.4-68.0$ & $28.9-34.0$ & $7.5-28.0$ & 17.0 & 10,23 \\
COVID-19 & 14.3 & 26.3 & 16.9 & 20.7 & 24,25 \\
\hline
\end{tabular}

COVID-19: coronavirus disease 2019; HD: heart disease; MERS: Middle East respiratory syndrome; SARS: severe acute respiratory syndrome.

mechanisms linking hyperglycemia and SARS-CoV-2 infection have been proposed including the regulation of the receptor expression used by the virus for cell entry, an altered immune response as well as an inflammatory echo.

Indeed, high blood glucose may increase viral entry and replication in vivo, possibly through the modulation of the ACE2 receptor [37-39]. We will further debate this aspect later in the review. Elevated glucose levels may also suppress the antiviral immune response increasing the severity of viral infections. Hyperglycemia affects the innate and adaptive immune responses at several levels: it reduces neutrophil degranulation, chemotaxis, and phagocytic activity; impairs complement activation; and inhibits lymphocyte proliferative response [40, 41]. As reported by Berbudi et al. hyperglycemia in diabetes is thought to cause dysfunction of the immune response which fails to control the spread of invading pathogens in diabetic subjects [42]. It has been reported that hyperglycemia strongly reduces the macrophagic activity of both neutrophils and macrophages exposing patients to infection [43]. During infection or inflammation, macrophages first exhibit the M1 phenotype to release TNF- $\alpha$, IL- $1 \beta$, IL-12, and IL- 6 against the stimulus. However, if the M1 phase persists, it can result in direct tissue damage. Therefore, M2 macrophages secrete high amounts of IL-10 and TGF- $\beta$ to suppress the inflammation, contribute to tissue repair, remodeling, vasculogenesis, and retain homeostasis [43]. In diabetes, M2 macrophages lose the capacity to suppress M1 macrophages activity promoting a chronic inflammation that makes diabetic subjects more susceptible to infections [43]. In addition, hyperglycemia activates Protein Kinase $\mathrm{C}$ inhibiting neutrophil migration, phagocytosis, superoxide production, and microbial killing. It can also induce Toll-like receptor expression inhibiting neutrophil functions and apoptosis [44]. As reported by Jafar et al. [44], high glucose concentrations decrease vascular dilation and increase permeability during the initial inflammatory responses, possibly through Protein Kinase C activation [44]. Furthermore, hyperglycemia can cause direct glycosylation of proteins and can alter the tertiary structure of complement; these changes inhibit immunoglobulin-mediated opsonization of bacteria and complement fixation to bacteria and decrease phagocytosis [44].

Previous evidence from other $\mathrm{CoV}$ models documented that MERS-CoV infection in a diabetic mouse model exhibited more severe disease and slower recovery due to delayed inflammation up to 21 days after infection [45]. Diabetic mice had fewer inflammatory monocyte/macrophages and CD4+ T cells which correlated with lower levels of TNF $\alpha$ and IL- 6 but higher levels of IL-17a expression. In line with these results, patients with SARS-CoV-2 are characterized by low peripheral CD4+ and CD8+ T cells counts, with high levels of proinflammatory Th17 CD $4+\mathrm{T}$ cells, as well as elevated cytokine levels $[15,46,47]$. Thus, these data suggest that the increased disease severity observed in individuals with SARS-CoV-2 infection and concomitant hyperglycemia is likely due to a dysregulated immune response, which results in more severe and prolonged pathology. Also, human studies have confirmed a strong correlation between hyperglycemia and worse outcome of SARS-CoV-2. Indeed, as reported by Bode et al. among 570 SARS-CoV-2 patients who died or were discharged, the mortality rate was $28.8 \%$ in 184 diabetes and/or uncontrolled hyperglycemia patients, compared with $6.2 \%$ of 386 patients without diabetes or hyperglycemia [48]. It is of interest that optimal glycemic control during hospitalization is associated with a reduction in risk of severe disease and death in patients with COVID19 , demonstrating that hyperglycemia remained a strong prognostic predictor of outcome in hospitalized patients with COVID-19. Furthermore, hyperglycemic COVID-19 patients versus normoglycemic ones displayed a higher cumulative incidence of severe disease [49]. Hyperglycemia may also affect pulmonary function, directly increasing glucose concentrations and vasculature permeability in airway secretions $[36,47]$ that facilitate the virus infection and replication [50]. A very recent work reports that COVID-19 patients with diabetes mellitus were more likely to have elevated inflammatory markers and hypercoagulability, accompanied by hypoproteinaemia and glucose and lipid metabolism disorders. As a consequent, COVID-19 combined with diabetes prolonged the time of detoxification in patients [51].

It is essential to establish a glycemic profile in SARS$\mathrm{CoV}-2$ patients to better control the clinical outcomes in patients with coexistent diabetes, obesity, and hyperglycemia. Obesity, as reported above, may represent another interesting factor associated with poor prognosis among COVID-19 patients. A complex cross talk between adipose tissue and the immune system may have a relevant implication for SARS-CoV-2 infection. In the physiological state, although the immune cells pervade adipose tissue, a balance between immune cells and adipocytes and, in turn, adipokines production is maintained [52]. In pathophysiological conditions, such as obesity, the adipose tissue is pervaded by an enormous number of immune cells leading to an imbalance in the production of adipokines such as adiponectin and leptin $[53,54]$. In addition, these adipokines are involved in energy homeostasis, glucose balance, and insulin sensitivity enhancing insulin sensitivity and glucose uptake and increasing, in turn, GLUT-4 translocation $[53,55]$. Understanding the mechanisms underlying interactions between adipocytes released hormones, immune 
deregulation, and comorbid related conditions may offer further insight into tracking the SARS-CoV-2 pathogenesis.

\subsection{ACE2 Receptor: Physiological Mechanisms and Implica-} tion for SARS-CoV-2 Infection. Coronaviruses attachment and adhesion to human target cells are mediated by a spike $(S)$ protein which protrudes from the viral surface $[56,57]$. For SARS-CoV and SARS-CoV-2 a binding of the S1 region, named receptor-binding domain (RBD), through the angiotensin-converting enzyme 2 (ACE2) receptor on host cells has been shown. The monocarboxypeptidase ACE2 was originally identified in 2000 as a homolog of ACE receptor $[58,59]$ and, subsequently, the molecular structure has been extensively described [60]. ACE2 expression is broadly represented in different biological systems including airway and type II lung alveolar cells, oesophageal epithelial cells, enterocytes, cholangiocytes, myocardial cells, kidney proximal tubule cells, and bladder urothelial cells [61]. In human lungs, ACE2 generates angiotensin (IVII) from angiotensin II by the cleavage of a single amino acid [62]. Ang-(I-VII) through Mas receptor (Mas1) activation is expressed on endothelial cells and results in vasodilatory, anti-inflammatory, and antifibrotic effects [63]. Interestingly, the interaction between SARS-CoV-2 and ACE2 induces the downregulation of ACE2 expression resulting in AngII accumulation with proinflammatory and profibrotic effects $[64,65]$. In a small cohort study, levels of Ang II were found markedly increased in SARS-CoV-2 plasma samples [66]. Elevated Ang II levels were found in mice infected with SARS [65] or H7N9 virus resulting in worse clinical outcomes $[57,67,68]$ Accumulation of AngII promotes cell apoptosis through the interaction with receptor AT1R [68]. Different signaling pathways may activate apoptotic stimuli. Firstly, exalted oxidative stress related to overactivation of the AngII/AT1R/NAPDHox axis has been correlated with cardiovascular disorders, mainly hypertension [69] and atherosclerosis [70]. Therefore, downstream generation of reactive oxygen species (ROS) promotes apoptosis through the release of CytC from damaged mitochondria [71], activation of caspase 3 [72], or p38MAPK/JNK cascade [73]. Furthermore, NF-kb activation and the transcription of cytokines such as interleukin-6, IL- $1 \beta$, and tumor necrosis factor alpha (TNF $\alpha$ ) have been also associated with proapoptotic signals [74]. High levels of AngII and proinflammatory cytokines can then act synergistically and increase the level of cyclooxigease 2 (COX2) with the consequent buildup of ROS and inflammatory prostaglandin E2 [75]. In hypertensive rats, low levels of ACE2 mRNA leading to reduced Ang-(I-VII)/Mas-1 signals have been documented as compared to nonhypertensive counterparts [76, 77]. Interestingly, polymorphisms for ACE2 were independently associated with increased hypertension susceptibility and cardiovascular complications in diabetes [76, 77]. The overactivation of these pathways can result in a state of hyperinflammation that is seen in the late phase of SARSCoV-2 infected patients.
3.3. ACE2 Expression Modulation: Implication for Antihypertensive and Antidiabetic Treatments. ACE2 expression is notably modified by agents acting on RAS. RAS inhibitors are the cornerstone of therapies for many cardiovascular and renal diseases. These drugs are widely used also in diabetic patients for preventing cardiovascular remodeling and diabetic nephropathy. Recently, in a multicenter retrospective study including hospitalized COVID-19 patients with previously diagnosed hypertension, the use of ACE inhibitors (ACE-Is) and Angiotensin Receptor Blockers (ARBs) was associated with lower all-cause mortality (adjusted HR, 0.42; 95\% CI, 0.19-0.92; $p=0.03$ ) and septic shock (adjusted HR, 0.36 ; 95\% CI, 0.16-0.84; $p=0.01$ ). Similarly, in another study of COVID-19 patients with hypertension, poor outcomes were witnessed when compared to nonhypertensive patients; interestingly, patients under ARBs/ACE-Is were less likely to be admitted to ICU (9.3\% vs. $22.9 ; p=0.061)$, with a lower death rate $(4.7 \%$ vs. $13.3 \% ; p=0.283)$ than treated with non-ACE-Is/ARBs agents, although these differences did not reach statistical significance [78].

Experimental and clinical models showed different responses to the administration of agents interfering with this regulatory axis: in particular ARBs and mineralocorticoidreceptor blockers seem to increase the levels of ACE2 expression [78, 79], while administration of ACE-Is, thus increasing cardiac ACE2 mRNA levels, did not result in enhanced ACE2 activity [79]. Early evidence of increased cardiac ACE2 expression was shown in the rat model. After myocardial infarction, the administration of ARBs (olmesartan or losartan) increased the ACE2 expression about threefold [80]. In another experimental model, losartan administration compensated ACE2 smoke-induced reduction therefore restoring the ACE/ACE2 ratio in the lung [81]. In another study, increased urinary ACE2 levels were observed in hypertensive patients treated with Olmesartan [82].

However, based on the above-discussed mechanisms linking ACE2 expression with local anti-inflammatory, antiproliferative, and antifibrotic proprieties, several international societies and associations have recommended against ACE-Is or sartans discontinuation in patients under chronic therapy [83].

Other antidiabetic agents potentially interfere with the regulation of the RAS. Thiazolidinediones are an important class of insulin sensitizers used in the treatment of type 2 diabetes. In diabetic patients, the molecular mechanisms of biological responses of thiazolidinediones are mediated through the modulation of Peroxisome Proliferators Activated Receptors (PPARs). Similar to ACEIs and ARBs, thiazolidinediones can upregulate ACE2 expression [84, 85] potentially exposing alveolar cells to SARS-CoV-2 infection. However, PPARs are mediators of inflammation with potential immunoregulatory characteristics. Their activation prompts a reduction in inflammatory cytokines that are highly involved in SARS-CoV-2 (i.e., IL6 and INF $\gamma$ ) [86]. Two different PPAR $\gamma$ agonists, rosiglitazone and pioglitazone, are insulin-mimetic drugs used in type 2 diabetes with high overall potential in reducing influenza virus infection [86]. In mice infected with the influenza virus, pioglitazone 


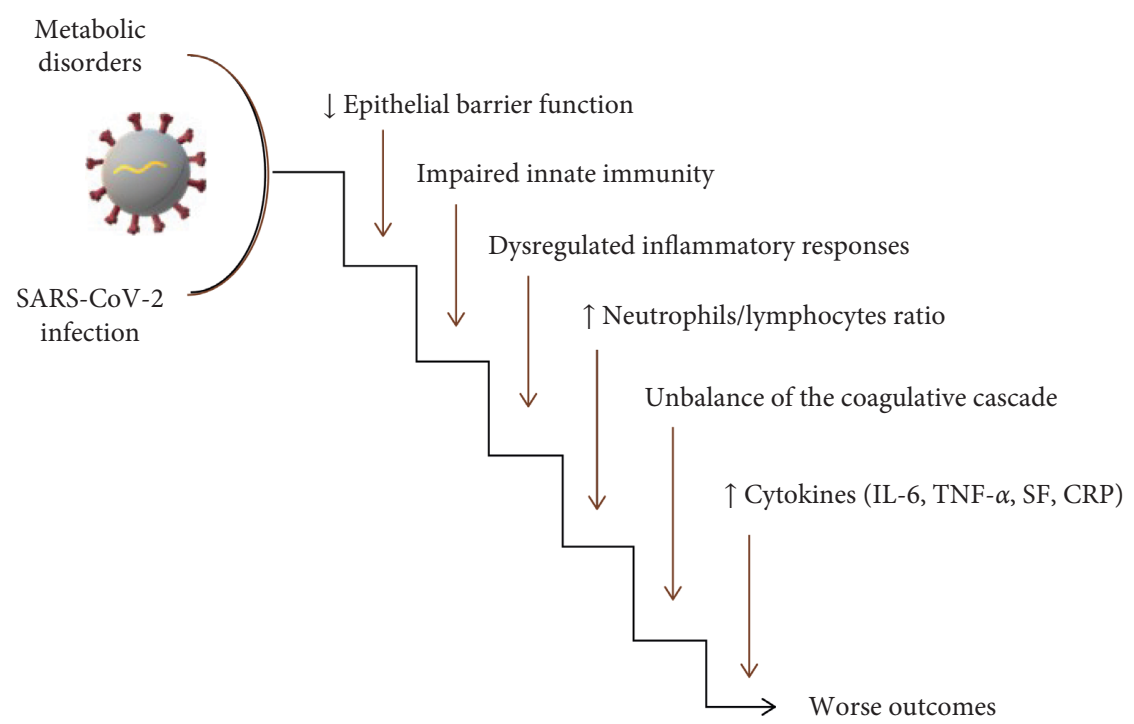

FIgURE 1: Molecular machineries responsible for the worsening of the SARS-CoV-2 phenotype and prognosis in patients with concomitant metabolic diseases. Biological functions such as inflammation, immunity, and epithelial defense mechanisms are involved.

administration resulted in improved survival based on antiinflammatory properties [86, 87].

\subsection{Increased Inflammation in SARS-CoV-2 Patients with} Metabolic Disorders. It is known that subjects affected by metabolic disorders, such as obesity and diabetes, have increased risk of bacterial, mycotic, parasitic, and viral infections [88]. Drucker et al. documented that acute viral respiratory infection has been linked to the rapid development of transient insulin resistance, in both otherwise healthy euglycemic normal weight or overweight individuals $[89,90]$, therefore demonstrating that infectious diseases strongly increase mortality in diabetic subjects. Retrospective analysis showed that mortality was increased in older diabetic patients [91]. Diabetic disease is strongly associated with increased risk and worse outcomes for bacterial and viral infections attributed to a combination of dysregulated innate immunity and inflammatory responses [92, 93]. Furthermore, coronavirus infections may be complicated by secondary bacterial infections, reflecting the compromised epithelial barrier function in the lungs and the gastrointestinal tract in diabetic subjects [89].

During viral infection, the chronic inflammation and related cytokines production induce neutrophilia, coagulation activation, and kidney injury leading to the death of patients with SARS-CoV-2 [94, 95]. Several studies have shown that, in diabetic patients with SARS-CoV-2, absolute count of lymphocytes in peripheral blood is significantly lower, while the absolute count of neutrophils is remarkably higher [96]. In addition, diabetic subjects with SARS-CoV-2 have higher serum levels of various inflammatory-related biomarkers compared to nondiabetic patients with SARSCoV-2 [57]. In particular, these subjects are characterized by elevated serum levels of IL- $6, \mathrm{TNF} \alpha$, serum ferritin, and C reactive protein (CRP). Among these, IL- 6 is a predictor of disease severity and prognosis and its expression time is longer than other cytokines (TNF $\alpha$ and IL-1) [97]. Furthermore, Guo et al. reported that, in diabetic patients, there is also an increase in serum ferritin indicating the activation of the monocyte-macrophage system, which is a crucial part of the inflammatory storm $[94,97]$. The authors concluded that patients with diabetes are susceptible to develop an inflammatory storm, which eventually leads to rapid deterioration of SARS-CoV-2 patients [97].

Finally, diabetes represents a noteworthy risk factor for impaired coagulative imbalance and platelet aggregation which might sustain the enhanced thromboembolic disorders observed in deceased patients with COVID-19 [98]. Several mechanisms linking inflammation and coagulative homeostasis in diabetic patients have been explored [95]. Firstly, D-dimer is increased by inflammation activating plasmin. Secondly, chronic inflammation and hypoxia activate thrombin and the activation of monocyte-macrophages causes secretion of a mass of tissue factors and activation of the exogenous coagulation pathway, which leads to an overall hypercoagulable state or even disseminated intravascular coagulation $[94,95]$. In addition, elevated D-dimer levels are consistently reported and their gradual increase during disease course is particularly associated with disease worsening [99]. In conclusion, during the disease course, longitudinal evaluation of lymphocyte count dynamics and inflammatory indices, such as CRP, IL-6, and ferritin may help to identify cases with poor prognosis and prompt intervention in order to improve outcomes.

3.5. Cytokine Role in Metabolic-Mediated Effects in SARSCoV-2. The uncontrolled production of IL-6 has been demonstrated to correlate with disease progression and severity [100], predicting respiratory failure in hospitalized symptomatic COVID-19 patients [101]. This could be accentuated in diabetic patients where low-grade inflammation, a characteristic of this pathology, can facilitate the 
cytokine storm caused by SARS-CoV-2 infection. Among the released cytokines, IL- 6 , IL- $1 \mathrm{~B}$, and TNF $\alpha$ can have a major impact on glucose metabolism, insulin signaling, and induce cardiovascular complications [102]. For instance, CRP can be stimulated by the release of IL-6 [103]. CRP can in turn aggregate and induce the activation of complement and tissue factor initiating coagulation [104]. It is worth noting that diabetic patients are more prone to develop coagulopathy such as disseminated intravascular coagulation (DIC) [105], a pathology that could be exacerbated in combination with SARS-CoV-2 infection and result in severe outcomes [106]. A further IL-6 increase in COVID-19 diabetic patients can exacerbate insulin resistance by inhibiting the autophosphorylation of the insulin receptor and the activation of PI3K and AKT pathway [107, 108]. Like IL-6, increased levels of TNF $\alpha$ have also been shown to induce insulin resistance by impairing insulin signaling and glucose uptake in vivo [109]. TNF $\alpha$ can induce the translocation of NF-kb into the nucleus and promote the transcription and release of more cytokines generating a vicious cycle. Finally, the overproduction of IL-1B can lead to B-cell dysfunction and death through the induction of nitric oxidase synthase and the excessive production of nitric oxide [110]. Leptin, a proinflammatory cytokine chronically elevated in obese patients, has been demonstrated to be a cofactor in the progression and severity of AH1N1 influenza leading to acute lung injuries [111] and thought to play a leading role in the development of insulin resistance, hypertension, and cardiovascular diseases [112]. Furthermore, the impaired immune response typical of obese patients can lead to poor vaccination success causing a delay in clearing the viral infection [113]. Given the impact that proinflammatory cytokines can have on glucose metabolism and insulin signaling, it is not surprising that diabetes represents a risk factor in SARS-CoV-2 infection. Furthermore, it may also be possible that a prolonged and acute increase of proinflammatory cytokines could predispose healthy subjects that have been critically affected by COVID-19, to develop diabetes.

\section{Conclusions}

SARS-CoV-2 patients with concomitant metabolic diseases are at higher risk of worse prognosis and mortality. Molecular mechanisms involving biological functions such as inflammation, immunity, and epithelial defense mechanisms may be implicated in exacerbating the infection leading to worsening clinical outcomes and even death (see Figure 1).

Interestingly, artificial intelligence and data mining approaches have been successfully used to determine the predictive biomarkers for the development of severe infection. An altered ACE2 expression, poor glycemic control, a mild upregulation of liver enzymes, alanine aminotransferase (ALT) and aspartate aminotransferase (AST), high interleukin 6, elevated hemoglobin, and lymphocytopenia are among the clinical presentation that predicts the development of severe COVID-19 infection $[14,114]$. This has led to examining the intricate relationship among pathophysiological pathways that may explain why diabetes is a risk factor for COVID-19 and link diabetes with liver disease. It is worth noting that elevated ALT has also been described as a predictor to develop diabetes [115]. Therefore, although clinical reports have shown that diabetic subjects are not at higher risk of SARS-CoV-2 infection compared to healthy subjects, if infected, they are more likely to develop a severe form [116-118]. This knowledge should be extremely useful to guide the clinical management of these patients.

An early diagnosis of metabolic and cardiovascular diseases must be ensured to optimize treatment. The relationship between coexisting metabolic diseases and unfavorable prognosis highlights the importance of rigorous glucose monitoring in COVID-19 patients. In addition, assessment of the coagulation axis and an evaluation of inflammatory markers such as CRP, IL-6, TNF $\alpha$, and serum ferritin, in metabolic patients infected with SARS-CoV-2, should be considered in the decision-making process. Lastly, although studies concerning the impact of antihypertensive and antidiabetic treatments upon COVID-19 course are still ongoing, medication adherence must be promoted to achieve complete control of underlying diseases and to better face the infection. As specific therapies for SARSCoV-2 are still not available, these considerations have major implications in public health especially for countries affected by a high incidence of metabolic disorders.

\section{Disclosure}

Ersilia Nigro and Fabio Perrotta contributed equally.

\section{Conflicts of Interest}

The authors declare no conflicts of interest.

\section{Authors' Contributions}

$\mathrm{EN}$ and $\mathrm{AD}$ convinced the study and wrote most of the manuscript. FP and VD discussed the role of ACE2. RP focused on the role of hyperglycemia. FS discussed the role of cytokines. $\mathrm{AD}$ and $\mathrm{AB}$ revised the manuscript. All authors approved the final version of the manuscript.

\section{Acknowledgments}

The authors thank Dr. Susan Campbell for revising and editing the text. This research received no external funding.

\section{References}

[1] C. Columbus, K. B. Brust, and A. C. Arroliga, "2019 novel coronavirus: an emerging global threat," Baylor University Medical Center Proceedings, vol. 33, no. 2, pp. 209-212, 2020.

[2] https://www.who.int/docs/default-source/coronaviruse/ situation-reports/20200414-sitrep-85-SARSCoV-2-19.pdf? sfvrsn=7b8629bb_4.

[3] http://www.epicentro.iss.it/coronavirus/bollettino/Infografic a_15aprile\%20ITA.pdf.

[4] T. Singhal, "A review of coronavirus disease-2019 (COVID19)," The Indian Journal of Pediatrics, vol. 87, no. 4, pp. 281-286, 2020. 
[5] A. C. Walls, Y.-J. Park, M. A. Tortorici, A. Wall, A. T. McGuire, and D. Veesler, "Structure, function, and antigenicity of the SARS-CoV-2 spike glycoprotein," Cell, vol. 181, no. 2, pp. 281-292, 2020.

[6] I. Ali and O. M. L. Alharbi, "COVID-19: disease, management, treatment, and social impact," Science of The Total Environment, vol. 728, Article ID 138861, 2020.

[7] L. Zhang, D. Lin, X. Sun et al., "Crystal structure of SARSCoV-2 main protease provides a basis for design of improved $\alpha$-ketoamide inhibitors," Science, vol. 368, no. 6489, pp. 409-412, 2020.

[8] D. Wang, B. Hu, C. Hu et al., "Clinical characteristics of 138 hospitalized patients with 2019 novel coronavirus-infected pneumonia in wuhan, China," JAMA, vol. 323, no. 11, p. 1061, 2020.

[9] H.-N. Gao, H.-Z. Lu, B. Cao et al., "Clinical findings in 111 cases of influenza A (H7N9) virus infection," New England Journal of Medicine, vol. 368, no. 24, pp. 2277-2285, 2013.

[10] F. Y. Alqahtani, F. S. Aleanizy, R. Mohamed et al., "Prevalence of comorbidities in cases of Middle East respiratory syndrome coronavirus: a retrospective study," Epidemiology and Infection, vol. 147, pp. 1-5, 2018.

[11] M. A. Garbati, S. F. Fagbo, V. J. Fang et al., "A comparative study of clinical presentation and risk factors for adverse outcome in patients hospitalised with acute respiratory disease due to MERS coronavirus or other causes," PLoS One, vol. 11, no. 11, Article ID e0165978, 2016.

[12] C. M. Rivers, M. S. Majumder, and E. T. Lofgren, "Risks of death and severe disease in patients with Middle East respiratory syndrome coronavirus, 2012-2015," American Journal of Epidemiology, vol. 184, no. 6, pp. 460-464, 2016.

[13] W.-J. Guan, W.-H. Liang, Y. Zhao et al., "Comorbidity and its impact on 1590 patients with covid-19 in China: a nationwide analysis," European Respiratory Journal, vol. 55, no. 5, Article ID 2000547, 2020.

[14] M. Marhl, V. Grubelnik, M. Magdič, and R. Markovič, "Diabetes and metabolic syndrome as risk factors for COVID-19," Diabetes \& Metabolic Syndrome: Clinical Research \& Reviews, vol. 14, no. 4, pp. 671-677, 2020.

[15] W.-J. Guan and N.-S. Zhong, "Clinical characteristics of covid-19 in China. Reply," The New England Journal of Medicine, vol. 382, 2020.

[16] G. Onder, G. Rezza, and S. Brusaferro, "Case-fatality rate and characteristics of patients dying in relation to COVID-19 in Italy," JAMA, vol. 323, no. 18, pp. 1775-1776, 2020.

[17] X. Yang, Y. Yu, J. Xu et al., "Clinical course and outcomes of critically ill patients with SARS-CoV-2 pneumonia in Wuhan, China: a single-centered, retrospective, observational study," Lancet Respiratory Medicine, vol. 8, no. 5, pp. 475-481, 2020.

[18] E. Nigro, R. Polito, A. Alfieri et al., "Molecular mechanisms involved in the positive effects of physical activity on coping with COVID-19," European Journal of Applied Physiology, vol. 120, no. 12, pp. 2569-2582, 2020 Sep 3.

[19] F. Grippo, S. Navarra, C. Orsi et al., "The role of COVID-19 in the death of SARS-CoV-2-positive patients: a study based on death certificates," Journal of Clinical Medicine, vol. 9, no. 11, Article ID E3459, 2020.

[20] N. Chen, M. Zhou, X. Dong et al., "Epidemiological and clinical characteristics of 99 cases of 2019 novel coronavirus pneumonia in Wuhan, China: a descriptive study," Lancet (London, England), vol. 395, no. 10223, pp. 507-513, 2020.

[21] C.-Y. Chen, C.-H. Lee, C.-Y. Liu, J.-H. Wang, L.-M. Wang, and R.-P. Perng, "Clinical features and outcomes of severe acute respiratory syndrome and predictive factors for acute respiratory distress syndrome," Journal of the Chinese Medical Association, vol. 68, no. 1, pp. 4-10, 2005.

[22] P. T. Tsui, M. L. Kwok, H. Yuen, and S. T. Lai, "Severe acute respiratory syndrome: clinical outcome and prognostic correlates," Emerging Infectious Diseases, vol. 9, no. 9, pp. 1064-1069, Sep. 2003.

[23] J. C. K. Chan, E. L. H. Tsui, and V. C. W. Wong, "Prognostication in severe acute respiratory syndrome: a retrospective time-course analysis of 1312 laboratory-confirmed patients in Hong Kong," Respirology, vol. 12, no. 4, pp. 531-542, 2007.

[24] C. M. Booth, L. M. Matukas, G. A. Tomlinson et al., "Clinical features and short-term outcomes of 144 patients with SARS in the greater Toronto area," JAMA, vol. 289, no. 21, pp. 2801-2809, 2003.

[25] C.-L. Liu, Y.-T. Lu, M.-J. Peng et al., "Clinical and laboratory features of severe acute respiratory syndrome vis-a-vis onset of fever," Chest, vol. 126, no. 2, pp. 509-517, 2004.

[26] A. Assiri, J. A. Al-Tawfiq, A. A. Al-Rabeeah et al., "Epidemiological, demographic, and clinical characteristics of 47 cases of Middle East respiratory syndrome coronavirus disease from Saudi Arabia: a descriptive study," The Lancet Infectious Diseases, vol. 13, no. 9, pp. 752-761, 2013.

[27] M. R. Mehra, S. S. Desai, S. Kuy, T. D. Henry, and A. N. Patel, "Cardiovascular disease, drug therapy, and mortality in covid-19," New England Journal of Medicine, vol. 382, no. 25, Article ID e102, 2020.

[28] J. Lighter, M. Phillips, S. Hochman et al., "Obesity in patients younger than 60 years is a risk factor for Covid-19 hospital admission," Clinical Infectious Diseases, vol. 71, no. 15, p. 896, 2020.

[29] J. W. M. Chan, C. K. Ng, Y. H. Chan et al., "Short term outcome and risk factors for adverse clinical outcomes in adults with severe acute respiratory syndrome (SARS)," Thorax, vol. 58, no. 8, pp. 686-689, 2003.

[30] https://www.epicentro.iss.it/en/coronavirus/sars-cov-2-anal ysis-of-deaths.

[31] B. Li, J. Yang, F. Zhao et al., "Prevalence and impact of cardiovascular metabolic diseases on COVID-19 in China," Clinical Research in Cardiology, vol. 109, no. 5, pp. 531-538, 2020.

[32] G. P. Fadini, M. L. Morieri, E. Longato, and A. Avogaro, "Prevalence and impact of diabetes among people infected with SARS-CoV-2," Journal of Endocrinological Investigation, vol. 43, no. 6, p. 867, 2020.

[33] Y. Sun, Q. Wang, G. Yang, C. Lin, Y. Zhang, and P. Yang, "Weight and prognosis for influenza A(H1N1)pdm09 infection during the pandemic period between 2009 and 2011: a systematic review of observational studies with metaanalysis," Infectious Diseases, vol. 48, no. 11-12, pp. 813-822, 2016.

[34] S. J. Carter, M. N. Baranauskas, and A. D. Fly, "Considerations for obesity, vitamin $\mathrm{D}$, and physical activity amidst the COVID-19 pandemic," Obesity, vol. 28, no. 7, p. 1176, 2020.

[35] A. Remuzzi and G. Remuzzi, "COVID-19 and Italy: what next?” Lancet (London, England), vol. 395, no. 10231, pp. 1225-1228, 2020.

[36] X. Li, S. Xu, M. Yu et al., "Risk factors for severity and mortality in adult COVID-19 inpatients in Wuhan," Journal of Allergy and Clinical Immunology, vol. 146, no. 1, p. 110, 2020. 
[37] K. D. Hulme, L. A. Gallo, and K. R. Short, "Influenza virus and glycemic variability in diabetes: a killer combination?" Frontiers in Microbiology, vol. 8, p. 861, 2017.

[38] J. Wysocki, M. Ye, M. J. Soler et al., "ACE and ACE2 activity in diabetic mice," Diabetes, vol. 55, no. 7, pp. 2132-2139, 2006.

[39] E. Longato, B. Di Camillo, G. Sparacino, C. Saccavini, A. Avogaro, and G. P. Fadini, "Diabetes diagnosis from administrative claims and estimation of the true prevalence of diabetes among 4.2 million individuals of the Veneto region (North East Italy)," Nutrition, Metabolism and Cardiovascular Diseases, vol. 30, no. 1, pp. 84-91, 2020.

[40] M. E. Stegenga, S. N. van der Crabben, R. M. E. Blümer et al., "Hyperglycemia enhances coagulation and reduces neutrophil degranulation, whereas hyperinsulinemia inhibits fibrinolysis during human endotoxemia," Blood, vol. 112, no. 1, pp. 82-89, 2008.

[41] M. P. Moutschen, A. J. Scheen, and P. J. Lefebvre, "Impaired immune responses in diabetes mellitus: analysis of the factors and mechanisms involved. Relevance to the increased susceptibility of diabetic patients to specific infections," Diabete Metab.vol. 18, no. 3, pp. 187-201, 1992.

[42] A. Berbudi, N. Rahmadika, A. I. Tjahjadi, and R. Ruslami, "Type 2 diabetes and its impact on the immune system," Current Diabetes Reviews, vol. 16, no. 5, p. 442, 2020.

[43] A. Shapouri-Moghaddam, S. Mohammadian, H. Vazini et al., "Macrophage plasticity, polarization, and function in health and disease," Journal of Cellular Physiology, vol. 233, no. 9, pp. 6425-6440, 2018.

[44] N. Jafar, H. Edriss, and K. Nugent, "The effect of short-term hyperglycemia on the innate immune system," The American Journal of the Medical Sciences, vol. 351, no. 2, pp. 201-211, 2016.

[45] K. A. Kulcsar, C. M. Coleman, S. E. Beck, and M. B. Frieman, "Comorbid diabetes results in immune dysregulation and enhanced disease severity following MERS-CoV infection," JCI Insight, vol. 4, no. 20, p. 20, 2019.

[46] C. Wu, X. Chen, Y. Cai et al., "Risk factors associated with acute respiratory distress syndrome and death in patients with coronavirus disease 2019 pneumonia in wuhan, China," JAMA Internal Medicine, vol. 180, no. 7, p. 934, 2020.

[47] Z. Xu, L. Shi, Y. Wang et al., "Pathological findings of COVID-19 associated with acute respiratory distress syndrome," The Lancet. Respiratory Medicine, vol. 8, no. 4, pp. 420-422, 2020.

[48] B. Bode, V. Garrett, J. Messler et al., "Glycemic characteristics and clinical outcomes of COVID-19 patients hospitalized in the United States," Journal of Diabetes Science and Technology, vol. 14, no. 4, pp. 813-821, Jul 2020.

[49] C. Sardu, N. D’Onofrio, M. L. Balestrieri, M. Barbieri et al., "Outcomes in patients with hyperglycemia affected by COVID-19: can we do more on glycemic control?" Diabetes Care, vol. 43, no. 7, pp. 1408-1415, 2020.

[50] B. J. Philips, J. Redman, A. Brennan et al., "Glucose in bronchial aspirates increases the risk of respiratory MRSA in intubated patients,” Thorax, vol. 60, no. 9, pp. 761-764, 2005.

[51] Y. Yan, F. Yang, X. Zhu et al., "Analysis of clinical features and pulmonary CT features of coronavirus disease 2019 (COVID-19) patients with diabetes mellitus," Endokrynologia Polska, vol. 71, no. 5, pp. 367-375, 2020.

[52] D. Popov and M. Simionescu, "Alterations of lung structure in experimental diabetes, and diabetes associated with hyperlipidaemia in hamsters," European Respiratory Journal, vol. 10, no. 8, pp. 1850-1858, 1997.
[53] M. A. Exley, L. Hand, D. O'Shea, and L. Lynch, "Interplay between the immune system and adipose tissue in obesity," Journal of Endocrinology, vol. 223, no. 2, pp. R41-R48, 2014.

[54] R. Polito, E. Nigro, A. Messina et al., "Adiponectin and orexin-A as a potential immunity link between adipose tissue and central nervous system," Frontiers in Physiology, vol. 9, p. 982, 2018.

[55] E. Nigro, P. Stiuso, M. G. Matera et al., “The anti-proliferative effects of adiponectin on human lung adenocarcinoma A549 cells and oxidative stress involvement," Pulmonary Pharmacology \& Therapeutics, vol. 55, p. 25, 2019.

[56] A. Pecoraro, E. Nigro, R. Polito et al., "Total and high molecular weight Adiponectin expression is decreased in patients with common variable immunodeficiency: correlation with ig replacement therapy," Frontiers in Immunology, vol. 8, p. 895, 2017.

[57] J. Yang, Y. Zheng, X. Gou et al., "Prevalence of comorbidities and its effects in patients infected with SARS-CoV-2: a systematic review and meta-analysis," International Journal of Infectious Diseases, vol. 94, pp. 91-95, 2020.

[58] S. R. Tipnis, N. M. Hooper, R. Hyde, E. Karran, G. Christie, and A. J. Turner, "A human homolog of angiotensin-converting enzyme," Journal of Biological Chemistry, vol. 275, no. 43 , pp. 33238-33243, 2000.

[59] M. Donoghue, F. Hsieh, E. Baronas et al., "A novel angiotensin-converting enzyme-related carboxypeptidase (ACE2) converts angiotensin I to angiotensin 1-9," Circulation Research, vol. 87, no. 5, pp. E1-E9, 2000.

[60] R. Yan, Y. Zhang, Y. Li, L. Xia, Y. Guo, and Q. Zhou, "Structural basis for the recognition of SARS-CoV-2 by fulllength human ACE2," Science, vol. 367, no. 6485, pp. 1444-1448, 2020.

[61] H. Xu, L. Zhong, J. Deng et al., "High expression of ACE2 receptor of 2019-nCoV on the epithelial cells of oral mucosa," International Journal of Oral Science, vol. 12, no. 1, p. 8, 2020.

[62] R. Gaddam, S. Chambers, and M. Bhatia, "ACE and ACE2 in inflammation: a tale of two enzymes," Inflammation \& $\mathrm{Al}$ lergy-Drug Targets, vol. 13, no. 4, pp. 224-234, 2014.

[63] A. R. Hemnes, A. Rathinasabapathy, E. A. Austin et al., "A potential therapeutic role for angiotensin-converting enzyme 2 in human pulmonary arterial hypertension," European Respiratory Journal, vol. 51, no. 6, Article ID 1702638, 2018.

[64] K. Kuba, Y. Imai, S. Rao et al., "A crucial role of angiotensin converting enzyme 2 (ACE2) in SARS coronavirus-induced lung injury," Nature Medicine, vol. 11, no. 8, pp. 875-879, 2005.

[65] Y. Imai, K. Kuba, S. Rao et al., "Angiotensin-converting enzyme 2 protects from severe acute lung failure," Nature, vol. 436, no. 7047, pp. 112-116, 2005.

[66] Y. Liu, Y. Yang, C. Zhang et al., "Clinical and biochemical indexes from 2019-nCoV infected patients linked to viral loads and lung injury," Science China Life Sciences, vol. 63, no. 3, pp. 364-374, 2020.

[67] P. Yang, H. Gu, Z. Zhao et al., “Angiotensin-converting enzyme 2 (ACE2) mediates influenza H7N9 virus-induced acute lung injury," Scientific Reports, vol. 4, no. 1, p. 7027, 2014.

[68] M. Papp, X. Li, J. Zhuang, R. Wang, and B. D. Uhal, “Angiotensin receptor subtype AT(1) mediates alveolar epithelial cell apoptosis in response to ANG II," American Journal of Physiology-Lung Cellular and Molecular Physiology, vol. 282, no. 4, pp. L713-L718, 2002. 
[69] A. Dikalova, R. Clempus, B. Lassègue et al., "Nox1 overexpression potentiates angiotensin II-induced hypertension and vascular smooth muscle hypertrophy in transgenic mice," Circulation, vol. 112, no. 17, pp. 2668-2676, 2005.

[70] E. S. Jones, A. Vinh, C. A. McCarthy, T. A. Gaspari, and R. E. Widdop, "AT2 receptors: functional relevance in cardiovascular disease," Pharmacology \& Therapeutics, vol. 120, no. 3, pp. 292-316, 2008.

[71] S. I. Dikalov and Z. Ungvari, "Role of mitochondrial oxidative stress in hypertension," American Journal of Physiology-Heart and Circulatory Physiology, vol. 305, no. 10, pp. H1417-H1427, 2013.

[72] D. Xue, Y. Li, Z. Jiang et al., "A ROS-dependent and Caspase3-mediated apoptosis in sheep bronchial epithelial cells in response to Mycoplasma Ovipneumoniae infections," Veterinary Immunology and Immunopathology, vol. 187, pp. 55-63, 2017.

[73] V. G. Cardoso, G. L. Gonçalves, J. M. Costa-Pessoa et al., "Angiotensin II-induced podocyte apoptosis is mediated by endoplasmic reticulum stress/PKC- $\delta / \mathrm{p} 38$ MAPK pathway activation and trough increased $\mathrm{Na}+\mathrm{H}+$ exchanger isoform 1 activity," BMC Nephrology, vol. 19, no. 1, p. 179, 2018.

[74] X. Zhang, M. Wu, H. Jiang et al., "Angiotensin II upregulates endothelial lipase expression via the NF-kappa B and MAPK signaling pathways," PLoS One, vol. 9, no. 9, Article ID e107634, 2014

[75] K. Kawahara, H. Hohjoh, T. Inazumi, S. Tsuchiya, and Y. Sugimoto, "Prostaglandin E2-induced inflammation: relevance of prostaglandin E receptors," Biochimica et Biophysica Acta (BBA) - Molecular and Cell Biology of Lipids, vol. 1851, no. 4, pp. 414-421, 2015.

[76] S. Keidar, A. Gamliel-Lazarovich, M. Kaplan et al., "Mineralocorticoid receptor blocker increases angiotensin-converting enzyme 2 activity in congestive heart failure patients," Circulation Research, vol. 97, no. 9, pp. 946-953, 2005.

[77] C. M. Ferrario, J. Jessup, M. C. Chappell et al., "Effect of angiotensin-converting enzyme inhibition and angiotensin II receptor blockers on cardiac angiotensin-converting enzyme 2," Circulation, vol. 111, no. 20, pp. 2605-2610, 2005.

[78] G. Yang, Z. Tan, L. Zhou et al., "Effects of angiotensin II receptor blockers and ACE (Angiotensin-Converting enzyme) inhibitors on virus infection, inflammatory status, and clinical outcomes in patients with COVID-19 and hypertension," Hypertension, vol. 76, no. 1, p. 51, 2020.

[79] J.-C. Zhong, J.-y. Ye, H.-y. Jin et al., "Telmisartan attenuates aortic hypertrophy in hypertensive rats by the modulation of ACE2 and profilin-1 expression," Regulatory Peptides, vol. 166, no. 1-3, pp. 90-97, 2011.

[80] Y. Ishiyama, P. E. Gallagher, D. B. Averill, E. A. Tallant, K. B. Brosnihan, and C. M. Ferrario, "Upregulation of angiotensin-converting enzyme 2 after myocardial infarction by blockade of angiotensin II receptors," Hypertension, vol. 43, no. 5, pp. 970-976, 2004.

[81] Y.-M. Yuan, L. Luo, Z. Guo, M. Yang, R.-S. Ye, and C. Luo, "Activation of renin-angiotensin-aldosterone system (RAAS) in the lung of smoking-induced pulmonary arterial hypertension (PAH) rats," Journal of the Renin-Angiotensin-Aldosterone System, vol. 16, no. 2, pp. 249-253, 2015.

[82] M. Furuhashi, N. Moniwa, T. Mita et al., "Urinary angiotensin-converting enzyme 2 in hypertensive patients may be increased by olmesartan, an angiotensin II receptor blocker," American Journal of Hypertension, vol. 28, no. 1, pp. 15-21, 2015.
[83] F. Perrotta, M. G. Matera, M. Cazzola, and A. Bianco, "Severe respiratory SARS-CoV2 infection: does ACE2 receptor matter?" Respiratory Medicine, vol. 168, no. 105996, 2020.

[84] R. M. Ali, M. Y. Al-Shorbagy, M. W. Helmy, and H. S. ElAbhar, "Role of Wnt4/ $\beta$-catenin, Ang II/TGF $\beta$, ACE2, NF$\kappa \mathrm{B}$, and IL-18 in attenuating renal ischemia/reperfusioninduced injury in rats treated with Vit D and pioglitazone," European Journal of Pharmacology, vol. 831, pp. 68-76, 2018.

[85] W. Zhang, C. Li, B. Liu et al., "Pioglitazone upregulates hepatic angiotensin converting enzyme 2 expression in rats with steatohepatitis," Annals of Hepatology, vol. 12, no. 6, pp. 892-900, 2013.

[86] I. Darwish, S. Mubareka, and W. C. Liles, "Immunomodulatory therapy for severe influenza," Expert Review of Antiinfective Therapy, vol. 9, no. 7, pp. 807-822, 2011.

[87] C. E. Moseley, R. G. Webster, and J. R. Aldridge, "Original Article: Peroxisome proliferator-activated receptor and AMP-activated protein kinase agonists protect against lethal influenza virus challenge in mice," Influenza and Other Respiratory Viruses, vol. 4, no. 5, pp. 307-311, 2010.

[88] A. Brufsky, "Hyperglycemia, hydroxychloroquine, and the COVID-19 pandemic," Journal of Medical Virology, vol. 92, no. 7 , p. 770,2020

[89] D. J. Drucker, "Coronavirus infections and type 2 diabetesshared pathways with therapeutic implications," Endocrine Reviews, vol. 41, no. 3, p. 457, 2020.

[90] M. Šestan, S. Marinović, I. Kavazović et al., "Virus-induced interferon- $\gamma$ causes insulin resistance in skeletal muscle and derails glycemic control in obesity," Immunity, vol. 49, no. 1, pp. 164-177, 2018.

[91] S. Rao Kondapally Seshasai, S. Kaptoge, A. Thompson et al., "Diabetes mellitus, fasting glucose, and risk of cause-specific death," New England Journal Medicine, vol. 364, no. 9, pp. 829-841, 2011.

[92] R. van Crevel, S. van de Vijver, and D. A. J. Moore, "The global diabetes epidemic: what does it mean for infectious diseases in tropical countries?" Lancet. Diabetes Endocrinology, vol. 5, no. 6, pp. 457-468, 2017.

[93] K. Hodgson, J. Morris, T. Bridson, B. Govan, C. Rush, and N. Ketheesan, "Immunological mechanisms contributing to the double burden of diabetes and intracellular bacterial infections," Immunology, vol. 144, no. 2, pp. 171-185, 2015.

[94] W. Guo, M. Li, Y. Dong et al., "Diabetes is a risk factor for the progression and prognosis of COVID -19," Diabetes/ Metabolism Research and Reviews, vol. 36, no. 7, Article ID e3319, 2020.

[95] M. Boccia, L. Aronne, B. Celia et al., "COVID-19 and coagulative axis: review of emerging aspects in a novel disease," Monaldi Archives for Chest Disease, vol. 90, no. 2, 2020.

[96] B. R. Shah and J. E. Hux, "Quantifying the risk of infectious diseases for people with diabetes," Diabetes Care, vol. 26, no. 2, pp. 510-513, 2003.

[97] R. Muniyappa and S. Gubbi, "COVID-19 pandemic, coronaviruses, and diabetes mellitus," American Journal of Physiology-Endocrinology and Metabolism, vol. 318, no. 5, pp. E736-E741, 2020.

[98] D. Wichmann, J.-P. Sperhake, M. Lütgehetmann et al., "Autopsy findings and venous thromboembolism in patients with COVID-19," Annals of Internal Medicine, vol. 173, no. 4, p. 268, 2020.

[99] E. Terpos, I. Ntanasis-Stathopoulos, I. Elalamy et al., "Hematological findings and complications of COVID -19," American Journal of Hematology, vol. 95, no. 7, p. 834, 2020. 
[100] B. Liu, M. Li, Z. Zhou, X. Guan, and Y. Xiang, "Can we use interleukin-6 (IL-6) blockade for coronavirus disease 2019 (COVID-19)-induced cytokine release syndrome (CRS)?" Journal of Autoimmunity, vol. 111, Article ID 102452, 2020.

[101] T. Herold et al., "Level of IL-6 predicts respiratory failure in hospitalized symptomatic COVID-19 patients," The Journal of Allergy and Clinical Immunology, vol. 146, no. 1, pp. 128-136, 2020.

[102] F. Perrotta, E. Nigro, M. Mollica et al., "Pulmonary hypertension and obesity: focus on adiponectin," International Journal of Molecular Sciences, vol. 20, no. 4, p. 912, 2019.

[103] S. Devaraj, U. Singh, and I. Jialal, "The evolving role of C-reactive protein in atherothrombosis," Clinical Chemistry, vol. 55, no. 2, pp. 229-238, 2009.

[104] W. P. Fay, "Linking inflammation and thrombosis: role of C-reactive protein," World Journal of Cardiology, vol. 2, no. 11, pp. 365-369, 2010.

[105] K. Nogami, I. Muraki, H. Imano, and H. Iso, "Risk of disseminated intravascular coagulation in patients with type 2 diabetes mellitus: retrospective cohort study," BMJ Open, vol. 7, no. 1, Article ID e013894, 2017.

[106] A. Kollias, K. G. Kyriakoulis, E. Dimakakos, G. Poulakou, G. S. Stergiou, and K. Syrigos, "Thromboembolic risk and anticoagulant therapy in COVID-19 patients: emerging evidence and call for action," British Journal of Haematology, vol. 189 , no. 5, p. 846, 2020.

[107] J. J. Senn, P. J. Klover, I. A. Nowak, and R. A. Mooney, "Interleukin-6 induces cellular insulin resistance in hepatocytes," Diabetes, vol. 51, no. 12, pp. 3391-3399, 2002.

[108] P. J. Klover, T. A. Zimmers, L. G. Koniaris, and R. A. Mooney, "Chronic exposure to interleukin-6 causes hepatic insulin resistance in mice," Diabetes, vol. 52, no. 11, pp. 2784-2789, 2003.

[109] P. Plomgaard, K. Bouzakri, R. Krogh-Madsen, B. Mittendorfer, J. R. Zierath, and B. K. Pedersen, "Tumor necrosis factor- induces skeletal muscle insulin resistance in healthy human subjects via inhibition of Akt substrate 160 phosphorylation," Diabetes, vol. 54, no. 10, pp. 2939-2945, 2005.

[110] C. A. Dinarello, "Blocking interleukin- $1 \beta$ in acute and chronic autoinflammatory diseases," Journal of Internal Medicine, vol. 269, no. 1, pp. 16-28, 2011.

[111] A. J. X. Zhang, K. K. W. To, C. Li et al., "Leptin mediates the pathogenesis of severe 2009 pandemic influenza A(H1N1) infection associated with cytokine dysregulation in mice with diet-induced obesity," The Journal of Infectious Diseases, vol. 207, no. 8, pp. 1270-1280, Apr. 2013.

[112] N. Hou and J.-D. Luo, "Leptin and cardiovascular diseases," Clinical and Experimental Pharmacology and Physiology, vol. 38, no. 12, pp. 905-913, 2011.

[113] E. A. Karlsson, T. Hertz, C. Johnson, A. Mehle, F. Krammer, and S. Schultz-Cherry, "Obesity outweighs protection conferred by adjuvanted influenza vaccination," MBio, vol. 7, no. $4,2016$.

[114] X. Jiang, M. Luo, Z. Zou, X. Wang, C. Chen, and J. Qiu, "Asymptomatic SARS-CoV-2 infected case with viral detection positive in stool but negative in nasopharyngeal samples lasts for 42 days," Journal of Medical Virology, vol. 92 , no. 10, p. 1807, 2020.

[115] N. Sattar, O. Scherbakova, I. Ford et al., "Elevated alanine aminotransferase predicts new-onset type 2 diabetes independently of classical risk factors, metabolic syndrome, and C-reactive protein in the West of Scotland Coronary
Prevention Study,” Diabetes, vol. 53, no. 11, pp. 2855-2860, 2004.

[116] L. Orioli, M. P. Hermans, J.-P. Thissen, D. Maiter, B. Vandeleene, and J.-C. Yombi, "COVID-19 in diabetic patients: related risks and specifics of management," Annales d'Endocrinologie, vol. 81, no. 2-3, pp. 101-109, 2020.

[117] A. Hussain, "Cardiac surgery and COVID-19: response to the letter to the editor," Journal Cardiac Surgery, vol. 35, no. $8,2020$.

[118] C. Huang, Y. Wang, X. Li et al., "Clinical features of patients infected with 2019 novel coronavirus in Wuhan, China," Lancet (London, England), vol. 395, no. 10223, pp. 497-506, 2020. 